\title{
Robotic computer system develops high-skill 'technosurgeons'
}

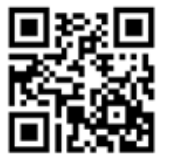

Just 15 months after four highly sophisticated robotassisted da Vinci Surgical Systems were introduced to South Africa (SA), nine local urologists are now 'flying' the ZAR23 million devices 'solo', while another ten are being mentored on the revolutionary robotic-assisted laparoscopic tool.

The surgical robotic system (four surgeon-controlled operating 'arms') was introduced via Earth Medical to four private hospitals in Cape Town, Johannesburg and Pretoria and gives highly skilled, appropriately trained specialists greater vision, precision and control during procedures, going beyond the flexibility and rotational capabilities of the human hand. It has also highlighted innate hospital/doctor and funder tensions in providing expensive but effective hightech medical equipment while maintaining viable patient funding. However, and perhaps most importantly, the da Vinci system has exposed an 'elephant in the room' - the internationally aberrant overuse by SA specialists of brachytherapy as a treatment modality for prostate cancer ( $73 \%$ above the global norm). Funded as a prescribed minimum benefit by medical aids (as is open prostatic surgery and traditional keyhole surgery), brachytherapy (the relatively quick and highly strategic placement of a radioactive 'pellet' at the prostate cancer site) allows urologists to conduct twice as many procedures a day as is possible with open or laparoscopic surgery.
Top academic compares treatments - and backs the device

Top academic and urologist Prof. André van der Merwe of Stellenbosch University conducted an in-depth comparative literature study of the available treatment modalities for prostatic cancer in SA. He concluded that brachytherapy should be reduced to international proportions (i.e. to $7 \%$ from the SA usage figure of $80 \%$ ), with robotic laparoscopic surgery a 'valuable tool' in helping achieve this. In his paper, he neutrally comments that should the caring physician benefit from one of the many options, then he might be biased in the manner he counsels the newly diagnosed patient', adding that a powerful differential exists between the urologist and the patient in consultations after a diagnosis of prostate cancer is made. Izindaba interviews with well-placed expert sources gleaned anecdotal evidence that some urologists often fail to discuss the pros and cons of various therapeutic options with their patients (most have very similar eventual outcomes), pushing brachytherapy and often not mentioning the sometimes appropriate strategy of 'watchful waiting' and active surveillance. Dr Jonathan Broomberg, CEO of SA's largest open medical aid, Discovery Health, said it was 'critical' that doctors discuss all available treatment options and the associated risks and benefits with their patients. Patient choice and decisions based on best evidence were 'vital if we are to achieve better value in our healthcare system'

Van der Merwe described robotic surgery in SA as 'a major step forward in patient care' and 'the beginning of a new chapter in local healthcare. He concluded that even though it is unfair to compare the early learning curve

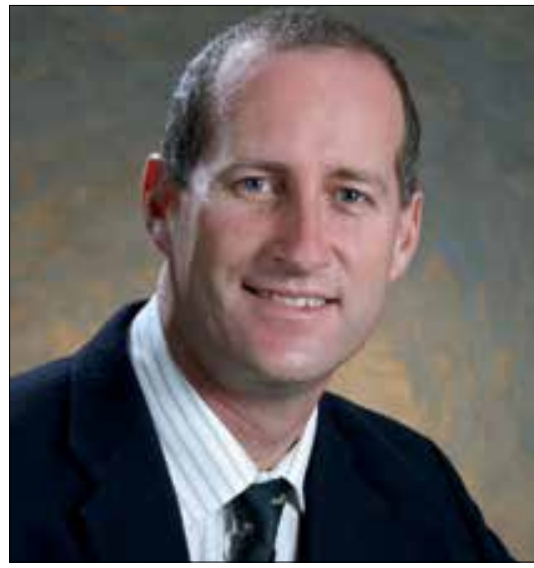

Prof. André van der Merwe.

of robotic prostatectomy with the established learning curve of open and laparoscopic radical prostatectomy, 'one could confidently say that the oncological (and urinary/sexual) outcomes are similar or better (using the da Vinci system), but definitely not worse'. Most importantly, properly performed, the camera-assisted robotic procedure is the international gold standard in minimally invasive surgery, halving recovery times and enabling unprecedented vision, precision and control (the 3-D HD camera image is magnified 10 times).

\section{As good as its operator}

The da Vinci, while an amazing piece of technology, is still a doctor-driven device. Training is taken very seriously, with doctors taught via a console-driven simulator where a $90 \%$ pass rate on 35 different test areas is required for hands-on proficiency. They are then sent to Belgium for live porcine practice. Following 
that, a highly experienced international urologist is flown out to SA to mentor the local urologists on patients via a 'buddy system. This involves two local urologists conducting a daily prostatectomy each for 10 days under supervision, the one local urologist observing and the other operating, and then swapping around in morning and afternoon sessions. This is obviously not teaching the urologist how to do a prostatectomy, but how to apply the da Vinci technology best to benefit from its advantages. The mentor may take over the console from time to time, just as a flying instructor would if potential safety concerns arise. Only when he is satisfied that they can use it safely and proficiently does he sign them off and they can go solo. Mentors insist that all procedures are filmed via the internal camera and e-mailed to them for ongoing monitoring and feedback, and they are continually available for advice and guidance. The average training time to full proficiency can vary from surgeon to surgeon. Simulator and initial local training takes some 45 hours and the overseas 'wet lab' training about 16 hours, while the local mentored stint typically covers 12 - 15 cases. Depending on the surgeon's skill levels and how quickly he or she gets the cases done, they may be spread out over between 3 months and a year, depending on a number of logistical factors. Newly signed-off urologists are counselled about the critical importance of patient selection, with obese patients initially avoided at all costs.

\section{Wider application imminent}

If the hospital and its doctors follow the proper surgery protocols, operations are safe, reproducible and result in reduced complications, minimal blood loss and improved functional and oncological outcome. According to Earth Medical, the rigorous training pathway eliminates any 'short cuts. Future applications for da Vinci surgery in SA would include appropriate colorectal and gynaecological procedures, and with over a year of successful use in prostatectomies, the intention is to move to other urological procedures including partial nephrectomies and and cystectomies.

One local hospital manager who has overseen the da Vinci introductory phase is Hein Calitz, general manager of Durbanville's Mediclinic Hospital. Quizzed on the health economics, Calitz said an entire operation cost about ZAR190 000 (including doctor and allied professionals' fees), of which they could recover only about ZAR125 000 - 135000 from some of the medical aids. However, certain medical aid schemes would only pay for open surgery and brachytherapy, flatly refusing to fund robotic surgery. 'At present we're writing that da Vinci medical aid payment shortfall off. We're not recovering from the patient yet. We're still crunching the numbers. It's for now more important to do volumes to get the surgeons fully trained than worry about getting a better price than ZAR125K and/or the balance in patient co-payments.' He intimated that over time, doctors would try and convince patients that it was worth their while to pay in the ZAR65 000 shortfall, although this could be phased in with patients paying half of this amount for the next year. Calitz said that the robot system was as susceptible to advancing technology as any other computer device, so that within $5-6$ years it would probably need replacing. His hospital group had built ZAR40 000 into the overall procedure price to recover some of the equipment expense. Calitz said when Mediclinic motivated for higher medical aid payouts for its robotic prostatectomies, the medical aids' response was that this would require an unrealistic increase in membership, or premium hikes, to make it financially viable. He said that punting procedure volumes, potentially improved surgical outcomes and vastly reduced patient recovery times cut no ice with medical aids, challenged as they already were by soaring new technology costs.

Broomberg told Izindaba that Discovery Health had performed a detailed health economic analysis on the use of the da Vinci robotic system for prostatectomy. Based on this, they funded the da Vinci robotic prostatectomy up to a 'defined rand limit', which was currently at a $40-60 \%$ premium over the cost of the open prostatectomy procedure. 'Our analysis indicates that this premium is the maximum that can be justified in terms of the incremental benefit of the da Vinci system, and also represents a sufficient reimbursement to the hospitals for the cost of the device. We've agreed a full reimbursement arrangement with some hospitals, but unfortunately, other hospitals insist on charging patients a higher rate, resulting in co-payments for some patients.'

Koert Pretorius, CEO of Mediclinic, said his company viewed the da Vinci system as research and development and a way of exposing doctors to the best research and technology while giving patients access to the latest treatment modalities. 'In principle we're prepared to make a contribution in the introductory phase, it's not just about money for us', he said.

\section{Chris Bateman}

chrisb@hmpg.co.za

S Afr Med J 2015;105(3):435-436.

DOI:10.7196/SAMJ.9464

\section{Case study: COPIC}

COPIC is a doctors' insurer based in Colorado, USA. They operate an in-house reporting system called the 'Three Rs' programme (Recognise, Respond, Resolve), where any doctor who has experienced an adverse outcome or had their patient express dissatisfaction with their care can contact a specific COPIC administrator to arrange their intervention. According to the COPIC website, the goals of the programme are 'to maintain the physician-patient relationship, facilitate open and honest communication and disclosure, and reimburse the patient for related out-of-pocket medical expenses.' ${ }^{\prime[1]}$

A series of interviews with patients who had participated in the programme revealed, tellingly, that: 'When the communication with the physician was good, open, and honest, the outcome was viewed as an honest mistake. Patients referred to their outcomes under these circumstances as both forgivable and understandable. Conversely, when the communication was perceived as poor or non-existent, the same outcome was viewed as an error or negligence.'[2]

Of the 1829 patients who had received reimbursement of their medical expenses through the programme, only $3.4 \%$ went on to make a claim against their doctor. While not officially described as a complaints system, COPIC's programme is an example of dissatisfaction handled effectively with a demonstrable effect on the likelihood of litigation.

1. www.callcopic.com/copic-services/safety-and-risk/Pages/3rs.aspx (accessed 7 October 2014).

2. Lembitz A. Litigation alternative: COPIC's 3Rs program. AAOS Now 2010;4(9). www.aaos.org/news/aaosnow/sep10/managing7.asp (accessed 5 May 2015). 